Sel ect i ve for mat i on of turbul ent struct ur es in magnet i zed cyl i ndr i cal pl asmas

\begin{tabular}{|l|l|}
\hline $\begin{array}{l}\text { jour nal or } \\
\text { publ i cat i on } \mathrm{ti} \text { t l e }\end{array}$ & Physi cs of Pl asmas \\
\hline vol une & Vol . 15 \\
\hline page r ange & 052302-1 - 052302-10 \\
\hline year & 2008- 05-01 \\
\hline URL & ht t p: //hdl . handl e. net /10655/2257 \\
\hline
\end{tabular}




\title{
Selective formation of turbulent structures in magnetized cylindrical plasmas
}

\author{
Naohiro Kasuya, ${ }^{1}$ Masatoshi Yagi, ${ }^{2}$ Kimitaka Itoh, ${ }^{1}$ and Sanae-I Itoh ${ }^{2}$ \\ ${ }^{1}$ National Institute for Fusion Science, Toki, Gifu 509-5292, Japan \\ ${ }^{2}$ Research Institute for Applied Mechanics, Kyushu University, Kasuga, Fukuoka 816-8580, Japan
}

(Received 12 September 2007; accepted 28 March 2008; published online 16 May 2008)

\begin{abstract}
The mechanism of nonlinear structural formation has been studied with a three-field reduced fluid model, which is extended to describe the resistive drift wave turbulence in magnetized cylindrical plasmas. In this model, ion-neutral collisions strongly stabilize the resistive drift wave, and the formed structure depends on the collision frequency. If the collision frequency is small, modulational coupling of unstable modes generates a zonal flow. On the other hand, if the collision frequency is large, a streamer, which is a localized vortex in the azimuthal direction, is formed. The structure is generated by nonlinear wave coupling and is sustained for a much longer duration than the drift wave oscillation period. This is a minimal model for analyzing the turbulent structural formation mechanism by mode coupling in cylindrical plasmas, and the competitive nature of structural formation is revealed. These turbulent structures affect particle transport. (C) 2008
\end{abstract} American Institute of Physics. [DOI: 10.1063/1.2912461]

\section{INTRODUCTION}

Turbulent plasmas form a variety of mesoscale structures such as a zonal flow and a streamer, ${ }^{1,2}$ which regulates microscale fluctuations and affects the level of the anomalous transport in fusion plasmas. Therefore, the formation and self-regulated mechanism of the turbulent structures should be taken into consideration to understand the transport processes. Drift wave instability is one of the candidates that induce anomalous transport in magnetically confined inhomogeneous plasmas. ${ }^{3}$ Plasma experiments in a simple linear configuration have been revisited recently for quantitative understandings of the structural formation mechanism by turbulence. ${ }^{4-12}$ A density gradient drives the drift wave turbulence in these plasmas. Two-dimensional measurements reveal the feature of turbulent structures ${ }^{13,14}$ and their formation mechanisms by nonlinear mode coupling. ${ }^{15,16}$ Numerical simulations of drift wave turbulence in linear devices have been carried out to understand the fundamental mechanism of structural formation by comparison with experiments. ${ }^{17-20}$

We have developed a three-dimensional numerical simulation code, which simulates the resistive drift wave turbulence in a linear device. ${ }^{17,18} \mathrm{~A}$ minimal model is solved to analyze the turbulent structural formation mechanism by mode coupling in cylindrical plasmas. The selective formation of the zonal flow and the streamer has been observed in the numerical simulation in a simple cylindrical configuration. We report the formation mechanism of the zonal flow and streamer and bifurcation of these turbulent structures using the ion-neutral collision frequency as a control parameter.

The paper is organized as follows: In Sec. II, the set of model equations for the analyses are described. Simulations using this model are explained in Sec. III. The nonlinear calculation gives formation of the zonal flow and streamer, and their formation mechanism is discussed in Sec. IV. The bifurcation of the turbulent structures using the ion-neutral collision frequency as a control parameter is presented in Sec. V, and the effect of structural formation on particle transport is discussed in Sec. VI. We summarize our results in Sec. VII. Numerical accuracy of simulation results is discussed in the Appendix.

\section{MODEL}

We have been developing a 3D numerical simulation code of the resistive drift wave turbulence in a linear device, called Numerical Linear Device (NLD) (details are described in Ref. 17). The three-field (density, potential, and parallel velocity of electrons) reduced fluid model is adopted. The plasma has a simple cylindrical shape, and the magnetic field has only the component in the axial direction with the uniform intensity. According to experiments, high density $\left[n_{e}\right.$ $\left.>1 \times 10^{19}\left(\mathrm{~m}^{-3}\right)\right]$ and low temperature $\left[T_{e}<5(\mathrm{eV})\right]$ plasmas in an argon discharge are analyzed. The density of neutral particles is high even in the plasma core region, ${ }^{21}$ so the effect of neutral particles is taken into consideration. The continuity equation, the vorticity equation, and Ohm's law can be used to obtain the fluctuating density, potential, and parallel velocity of electrons, ${ }^{22}$

$$
\begin{aligned}
& \frac{d N}{d t}=-\nabla_{\|} V-V \nabla_{\|} N+\mu_{N} \nabla_{\perp}^{2} N+S_{N}, \\
& \frac{d \nabla_{\perp}^{2} \phi}{d t}=\nabla N \cdot\left(-\nu_{\text {in }} \nabla_{\perp} \phi-\frac{d \nabla_{\perp} \phi}{d t}\right)-\nu_{\text {in }} \nabla_{\perp}^{2} \phi-\nabla_{\|} V \\
& -V \nabla_{\|} N+\mu_{W} \nabla_{\perp}^{4} \phi \text {, } \\
& \frac{d V}{d t}=\frac{M}{m_{e}}\left(\nabla_{\|} \phi-\nabla_{\|} N\right)-\nu_{e} V+\mu_{V} \nabla_{\perp}^{2} V,
\end{aligned}
$$

where $N=\ln \left(n / n_{0}\right), V=v_{\|} / c_{s}, \phi=e \varphi / T_{e}, n$ is the density, $n_{0}$ is the density at $r=0, v_{\|}$is the electron velocity parallel to the 
magnetic field, $c_{s}$ is the ion sound velocity, $\varphi$ is the electrostatic potential, $T_{\mathrm{e}}$ is the electron temperature, $d / d t=\partial / \partial t$ $+[\phi$,$] is the convective derivative, S_{N}$ is a particle source term, $M / m_{e}$ is mass ratio of the ion and electron, $\nu_{\text {in }}$ is the ion-neutral collision frequency, $\nu_{e}=\nu_{e i}+\nu_{e n}$ is the sum of the ion-electron and electron-neutral collision frequency, and $\mu_{N}$, $\mu_{V}$, and $\mu_{W}$ are artificial viscosities. The ion cyclotron frequency $\Omega_{c i}$ and Larmor radius measured by the electron temperature $\rho_{s}$ are used for the normalizations. The equations are solved in the cylindrical coordinate with spectral expansion in the azimuthal and axial directions assuming periodic boundary condition, where $m$ and $n$ are the azimuthal and axial mode number, respectively. The boundary condition in the radial direction are set to $f=0$ at $r=0, a$ when $m \neq 0$, and $\partial f / \partial r=0$ at $r=0, f=0$ at $r=a$ when $m=0$, where $f$ implies $\{N, \phi, V\}$, and $r=a$ gives an outer boundary of the plasma column.

\section{NONLINEAR SIMULATION}

\section{A. Simulation parameters}

A nonlinear simulation has been performed to examine the saturation mechanism of the resistive drift wave turbulence. The following parameters are used: $B=0.1(\mathrm{~T}), T_{e}$ $=2(\mathrm{eV}), a=10(\mathrm{~cm})$, length of the device $\lambda=1.7(\mathrm{~m}), \mu_{N}$ $=1 \times 10^{-2}$, and $\mu_{V}=\mu_{W}=1 \times 10^{-4}$. Using these parameters, $\nu_{e}$ is estimated to be $\nu_{e}=310 .{ }^{17}$ The electron collisions $\left(\nu_{e i}\right.$ and $\left.\nu_{e n}\right)$ destabilize and the ion-neutral collisions $\left(\nu_{\text {in }}\right)$ stabilize the resistive drift wave. ${ }^{17}$ Therefore, the drift wave can be excited with large $\nu_{e}$ and small $\nu_{\text {in }}$. There is ambiguity of the absolute value of collision frequency $\nu_{\mathrm{in}}$, which depends on the neutral density. Therefore, $\nu_{\text {in }}$ is used as a parameter for controlling the instability in our simulations.

The calculation with a fixed particle source has been carried out, where the time independent source profile is given by

$$
S_{N}(r)=\frac{4 S_{0} \mu_{N}}{L_{N}^{2}}\left[1-\left(\frac{r}{L_{N}}\right)^{2}\right] \exp \left[-\left(\frac{r}{L_{N}}\right)^{2}\right],
$$

with $S_{0}=5.0, L_{N}=5(\mathrm{~cm})$. A density profile peaked at $r=0$ is formed by this particle source, and destabilizes the resistive drift wave.

\section{B. Linear-growth phase}

Using the parameters described in the previous subsection, simulations are carried out. Simulations in this paper are performed with 256 grids in the radial direction. In the azimuthal and axial directions, the Fourier expansion is adopted, and Fourier modes $(m, n)=(0,0)$ and $m$ $= \pm 1- \pm 16, n= \pm 1- \pm 16$ are kept $(m \times n=16 \times 16)$. The initial condition is given to be $f=0$ for $(m, n)=(0,0)$ and $f$ $=1 \times 10^{-8} \sin (\pi r / a)$ for all the other modes, where $f$ implies $\{N, \phi, V\}$, i.e., $n \neq 0$ perturbations are added in the initial state. The time evolution of each mode is calculated with Eqs. (1)-(3). Numerical accuracy has been verified by comparing calculations with different numbers of grids and Fourier modes (see the Appendix). Most unstable modes $(3,1)$

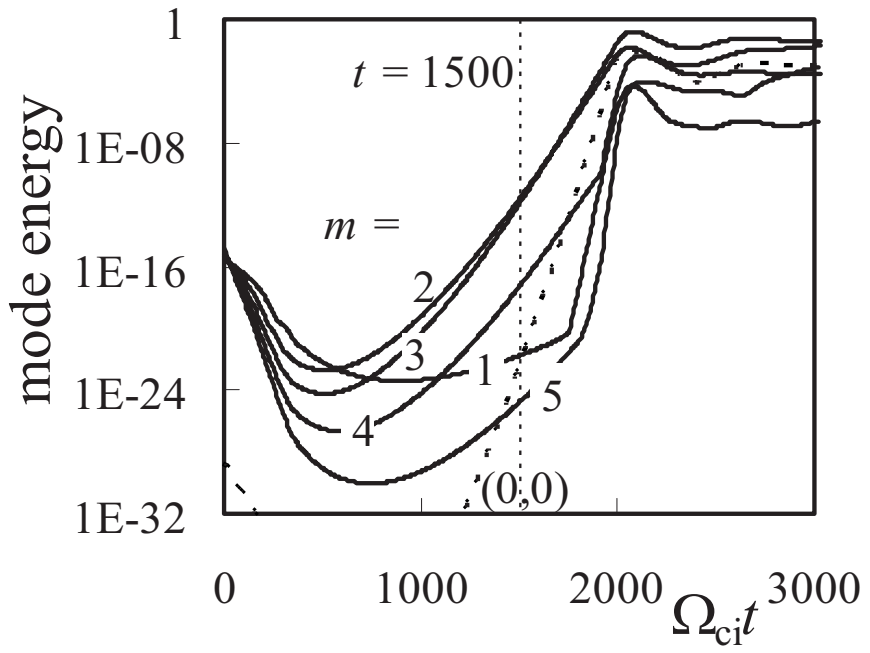

FIG. 1. Time evolution of the potential energy of each Fourier mode in the case with $\nu_{\text {in }}=0.1$.

and $(2,1)$ linearly increase their amplitudes, as shown in Fig. 1. Figure 2 shows linear growth rates and eigenfrequencies. The solid lines indicate instantaneous growth rates with the density profile at $t=1500$ and $\phi_{0}=0$. The closed circles indicate those evaluated by fitting the energy evolution of each Fourier mode at $t=1500$. Good agreement is obtained for both evaluation methods of the linear growth rate. It is found that only $n=1$ modes are unstable and $n>1$ modes are all stable with these parameters.

\section{Nonlinear saturation}

The nonlinear coupling excites the other modes after the linear phase. It is clearly seen that the $(1,1)$ mode is nonlinearly accelerated after $t=1760$ in Fig. 1 . This is due to the incoherent emission from high $m$ modes and is explained by the weak turbulence theory. ${ }^{23}$ The $(0,0)$ mode is also gener-

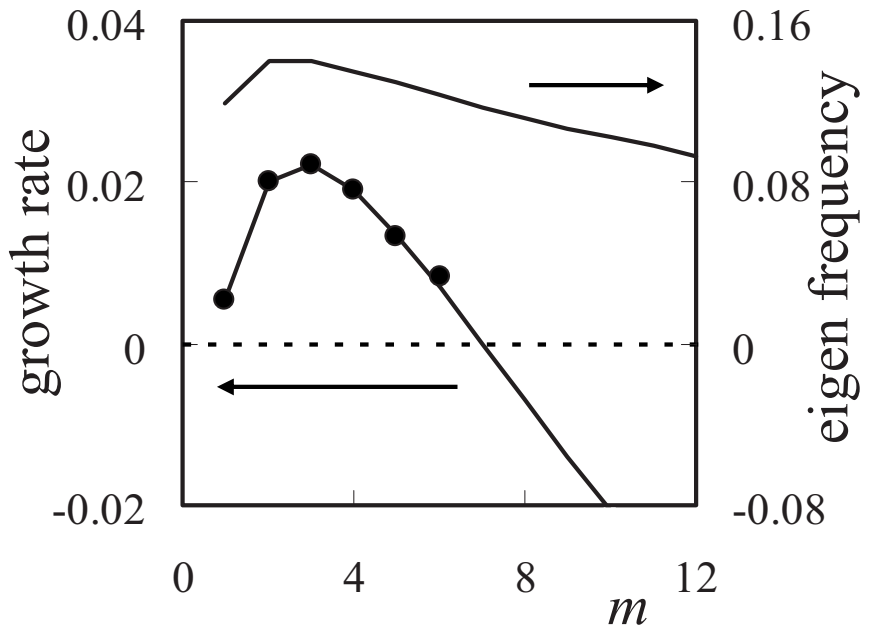

FIG. 2. Linear growth rate and eigenfrequency of mode $n=1$. Dependencies on the azimuthal mode number $m$ in the case with $\nu_{\text {in }}=0.1$ are shown. Solid lines indicate instantaneous growth rates calculated with the density profile at $t=1500$ and $\phi_{0}=0$, and the closed circles indicate those evaluated by fitting the evolution of each Fourier mode at $t=1500$ in Fig. 1 . The values are normalized by $\Omega_{c i}$. 


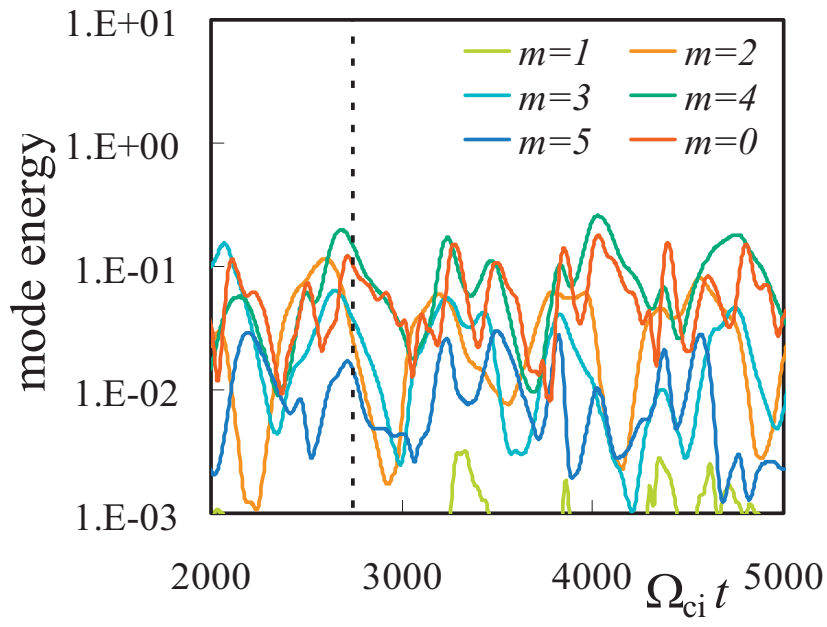

FIG. 3. (Color) Time evolutions of Fourier modes of the fluctuation energy in the nonlinear saturation stage. The fluctuation energies of the electrostatic potential of modes $(m, n)=(0,0),(1-5,1)$ are shown when $\nu_{\text {in }}=0.02$.

ated, and the density flattening occurs in the core region $(0.1<r / a<0.7)$, which is attributed to an enhanced transport owing to the fluctuations. This quasilinear effect and the stabilization by the mean potential give the mode saturation. The details of these saturation mechanisms are described in Ref. 17. Mechanism of nonlinear excitation of the zonal flows and streamers can be studied by analyzing this turbulence.

\section{TURBULENT STRUCTURES}

Turbulent structure is formed in the nonlinear saturation phase, which depends on the ion-neutral collision frequency $\nu_{\text {in. }}$. It has two effects: One is the damping force of the zonal flow and the other is the driving force of the drift wave instability. ${ }^{17}$ The former effect appears in the time evolution of $\phi_{00}$ obtained from Eq. (2),

$$
\begin{aligned}
\frac{1}{2} \frac{\partial}{\partial t}\left\langle\left|\frac{\partial \phi_{00}}{\partial r}\right|^{2}\right\rangle= & \left\langle\phi_{00}\left[\phi, \nabla_{\perp}^{2} \phi\right]_{00}\right\rangle \\
& +\nu_{\text {in }}\left\langle\phi_{00}\left(\nabla_{\perp} N \cdot \nabla_{\perp} \phi\right)_{00}\right\rangle \\
& +\left\langle\phi_{00}\left(\nabla_{\perp} N \cdot\left[\phi, \nabla_{\perp} \phi\right]\right)_{00}\right\rangle \\
& +\left\langle\phi_{00}\left(V \nabla_{\|} N\right)_{00}\right\rangle-\nu_{\text {in }}\left\langle\left|\frac{\partial \phi_{00}}{\partial r}\right|^{2}\right\rangle \\
& -\mu_{W}\left\langle\left(\nabla_{\perp}^{2} \phi_{00}\right)^{2}\right\rangle,
\end{aligned}
$$

where \langle\rangle represents the flux surface average. The fifth term in the right-hand side of the equation is the damping term by neutral collisions. These two effects together dictate the formation of the turbulent structure and the level of fluctuations in nonlinear states.

\section{A. Zonal flow formation}

If the collision frequency is small, compared with the growth rate of unstable modes, modulational coupling of unstable modes generates the $(0,0)$ mode. Figure 3 shows the time evolution of the fluctuation energy of the potential, de- composed into Fourier components, in the case with $\nu_{\text {in }}$ $=0.02$. It is seen that modes with $(m, n)=(2-5,1)$ have the same level of the amplitude with a bursty oscillation. The mechanism of the oscillation is as follows: First, unstable modes with $(m, n)=(2-4,1)$ increase. Then, nonlinear coupling $(m, n)+(-m,-n) \rightarrow(0,0)$ causes the growth of the $(0$, $0)$ mode. The mode with the largest amplitude, which varies every moment, contributes most to the $(0,0)$ mode generation. The generated mean potential stabilizes the unstable modes, which turn to decrease. Without the nonlinear source, the $(0,0)$ mode cannot be sustained, and once the mean potential becomes small enough, the $m \neq 0$ modes begin to grow again. In this way, the limit cycle oscillation is generated (as was reviewed in Ref. 1).

Figure 4 shows the contour plots of the electrostatic potential at $t=2740$. The top figure corresponds to the one in the $\theta-z$ plane at $r / a=0.5$ and the bottom figures to those in the $r$ - $\theta$ plane at $z / \lambda=0.2,0.3,0.4$, and 0.8 , respectively. In the axial direction, the structure extends in the direction parallel to the magnetic field with axial mode number $n=1$. Modes with $m \neq 0$ and $n=0$ (homogeneous in the axial direction) are not solved here, and turbulent structures discussed in this paper have nodes in the axial direction.

\section{B. Streamer formation}

If the collision frequency is large, the zonal flow remains stable, owing to strong collisional damping. Parametric coupling with modes, which have neighboring $m$ and a frequency close to each other, forms a streamer. Figure 5 shows the time evolution of the fluctuation energy of the potential in the case with $\nu_{\text {in }}=0.1$. Modes with $(m, n)=( \pm 4, \pm 1)$ and $( \pm 5, \pm 1)$ are dominant in this case. The mode with a maximum amplitude changes from time to time, and evolutions of the mode amplitudes are rather periodic compared with those in the small $\nu_{\text {in }}$ case in the previous subsection. These modes have frequencies close to each other $[|\omega(5,1)-\omega(4,1)|$ $\sim 0.01|\omega(5,1)|]$, and couple with each other by the mediator mode $(1,2)$ to form a streamer. Figure 6 shows snapshots of the contour plot of the electrostatic potential. A strong vortex is localized in the $\theta$ direction, and rotates in the electron diamagnetic direction. In the axial direction, the structure extends in the direction parallel to the magnetic field with axial mode number $n=1$, which is the same in the small $\nu_{\text {in }}$ case. Figure 7 shows a time evolution of the $\theta$ profile of the radial $\mathrm{E} \times \mathrm{B}$ convective velocity. The localized structure, streamer, induces localized convective transport in the radial direction as shown in Fig. 7.

Here, the streamers are characterized to be (i) their typical scale $k_{\theta}$ : Finite, $k_{r} \sim k_{z} \sim 0, \omega \ll \omega$ (microturbulence), (ii) formed by nonlinear processes, i.e., three wave coupling, and (iii) localized in the direction of wave propagation $(\theta$ direction). ${ }^{1}$ A localized structure in the $\theta$ direction needs several Fourier modes to be constructed.

The streamer in our simulation consists of rather small number of dominant contributors, $(4,1)$ and $(5,1)$, which are coupled with each other by means of the mediator mode (1, 2 ), and form a quasimodelike structure. On the other hand, the typical scale length of the drift wave $\rho_{i}$ is not so small 


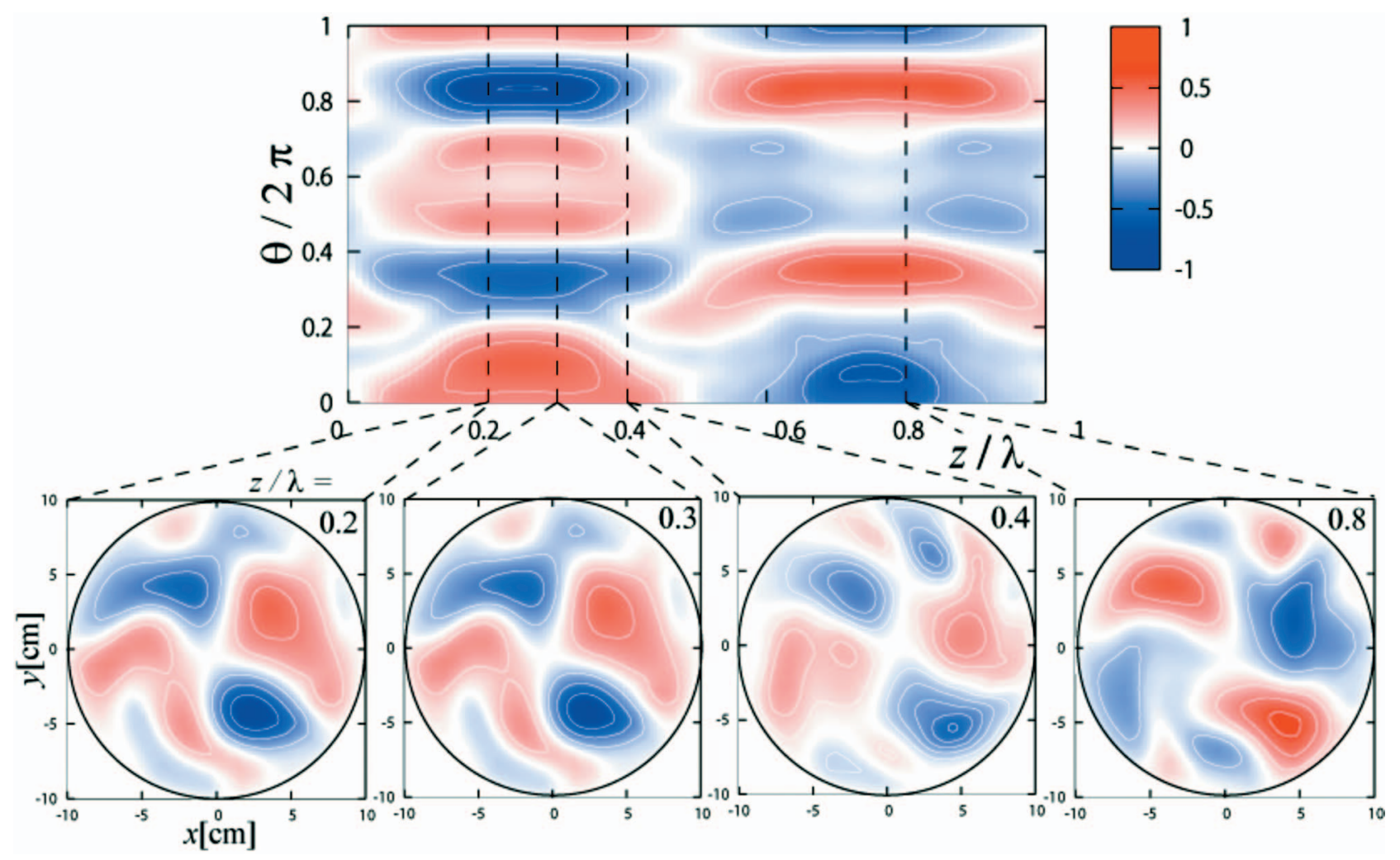

FIG. 4. (Color) Contour plots of the electrostatic potential at $r / a=0.5$ in the $\theta-z$ plane, and at $z / \lambda=0.2,0.3,0.4$, and 0.8 in the $r-\theta$ plane. These are snapshots at $t=2740$, which is indicated by the dashed line in Fig. 3 .

compared with the plasma radius $a\left(\rho_{i} / a \sim 0.09\right)$. Therefore, the turbulent structure discussed here has a scale length close to the plasma radius. However, the rotation frequency of the turbulent structure is $f_{\mathrm{st}}=3 \times 10^{-4}$, which is much smaller than that of the drift wave, e.g., that of the $(4,1)$ mode $f_{4,1}$ $=0.02\left(f_{4,1} / f_{\mathrm{st}} \sim 70\right)$. In this way, the characteristics of streamers are satisfied without the typical space length, and we call the structure a streamer.

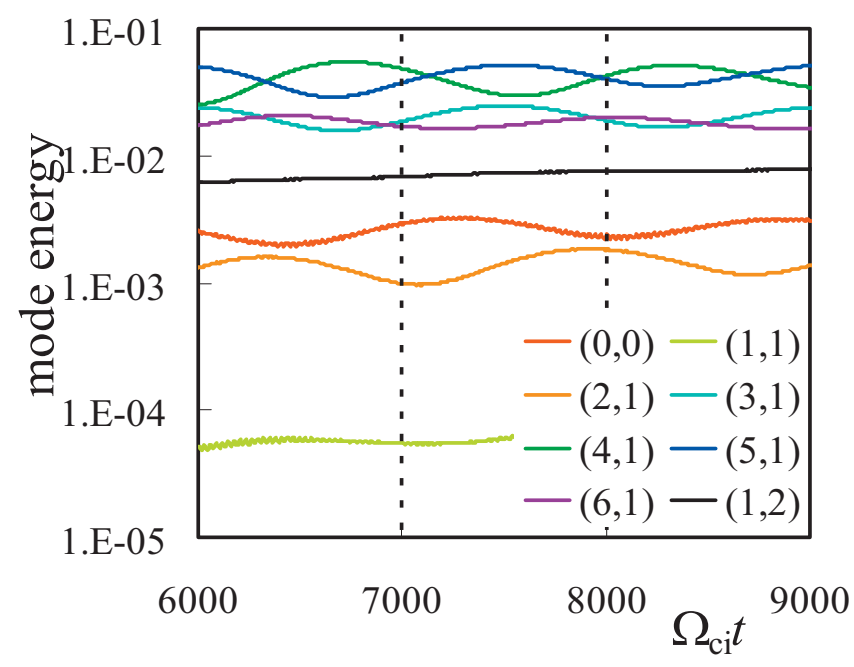

FIG. 5. (Color) Time evolutions of Fourier modes of the fluctuation energy in the nonlinear saturation stage. The fluctuation energies of the electrostatic potential of modes $(m, n)=(0,0),(1-6,1),(1,2)$ are shown when $\nu_{\text {in }}=0.1$.

\section{Mechanism of streamer formation}

A vortex structure localized in the $\theta$ direction, shown in Fig. 6, is sustained for a much longer duration than the drift wave oscillation period (more than $3000 \Omega_{c i}$ in Fig. 7). This mode matching comes from three wave coupling.

Figure 8 shows dependencies of the instantaneous linear growth rate and eigenfrequency on the azimuthal mode number $m$ in the case of $n=1$ and $\nu_{\text {in }}=0.1$. They are evaluated with the density and potential profile at $t=8000$. The $(6,1)$ mode has a maximum growth rate, though the $(4,1)$ or $(5,1)$ mode has the largest amplitude in the nonlinear saturated stage, as shown in Fig. 5. It implies that the nonlinear effect is more prominent. Figure 9 shows angular frequencies in the saturation phase $\omega_{N}$ and linear eigenfrequencies $\omega_{L}$ of the $(4$, $1)$ and $(5,1)$ mode. Both frequencies of the modes $(4,1)$ and $(5,1)$ are downward shifted by nonlinear effects, and become closer to each other when the three wave coupling [shown in Fig. 10(b)] is strong. In the zonal flow case, large variation of the frequency takes place by means of the potential oscillation.

To clarify the mechanism, the rate of the energy transfer of each mode $d E(m, n) / d t$ is calculated. Equations (1)-(3) can be divided into two parts: The part proportional to the own mode energy, and the other nonlinear coupling part. Linear (LT) and nonlinear (NT) contributions in $d E(m, n) / d t$ are calculated. Figures 10(a) and 10(b) show the time evolution of the mode energy and the sum of the nonlinear energy transfer to the $(4,1)$ mode, respectively. The magnitude of 
(a) $t=7000$
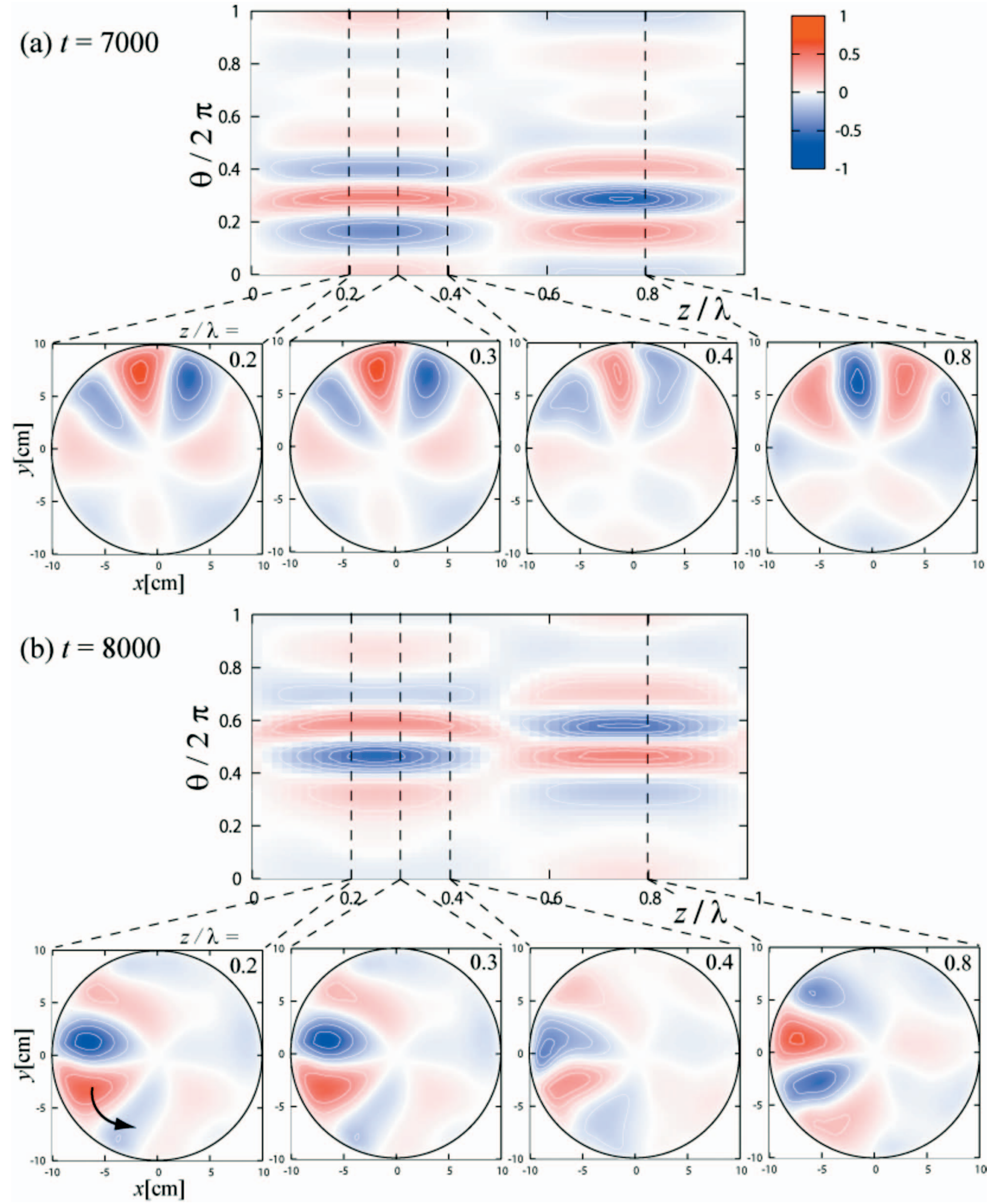

FIG. 6. (Color) Contour plots of the electrostatic potential at $r / a=0.5$ in the $\theta-z$ plane, and at $z / \lambda=0.2,0.3,0.4$, and 0.8 in the $r$ - $\theta$ plane. These are snapshots at (a) $t=7000$ and (b) $t=8000$, which are indicated by the dashed lines in Fig. 5.

the NT is comparable to the LT, but has a different sign. It indicates that the LT drives the limit cycle oscillation of modes, and on the other hand, NT suppresses it. To clarify which mode couplings are dominant in nonlinear energy transfer, NT is decomposed into components, corresponding to each three-wave coupling [Fig. 10(c)]. Couplings with neighboring modes, such as $(4,1) \leftarrow(3,-1)+(1,2)$ or $(5,-1)+(-1,2)$, are dominant. Although the amplitude of $(3,-1)+(1,2)$ coupling is largest, it is almost cancelled out by the LT, as the result, the total sum almost agrees with the contribution from $(5,-1)+(-1,2)$ coupling, which is most important for streamer formation in this case. If the mediate 


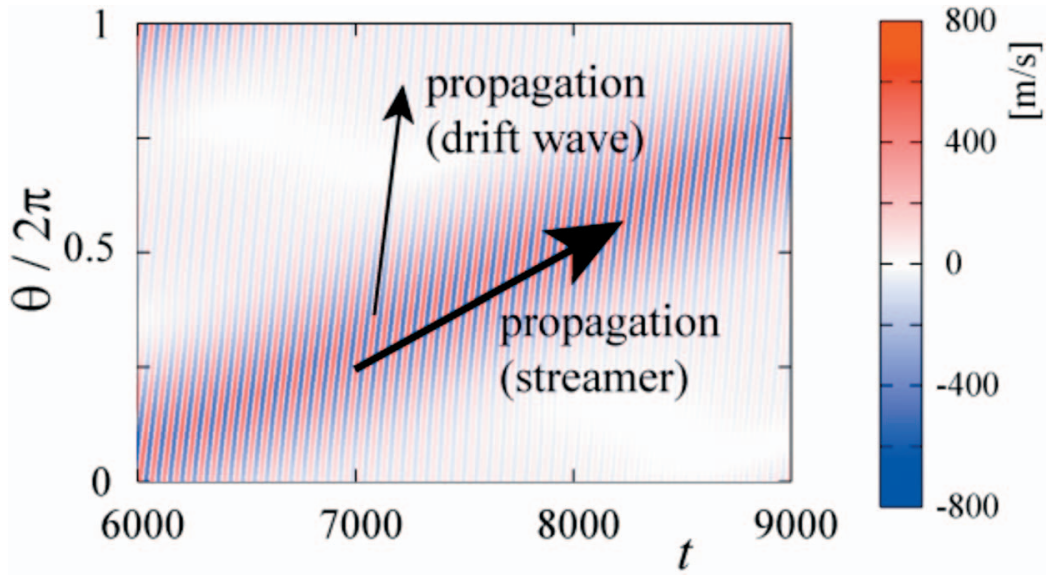

FIG. 7. (Color) Time evolution of the $\theta$ profile of the convective velocity at $r / a=0.5$ and $z / \lambda=0.375$. mode $(1,2)$ is artificially removed after the saturation, the streamer is not sustained and only a single mode becomes dominant. These results suggest the important role of the mediator mode for the streamer formation.

\section{SELECTIVE STRUCTURAL FORMATION}

Selective formation of the turbulent structure can be identified by changing a damping parameter. Nonlinear simulations are carried out by changing the ion-neutral collision frequency from $\nu_{\text {in }}=0.01$ to 0.12 . Figure 11 shows the dependencies of averaged fluctuation energies of the potential on $\nu_{\text {in }}$. These are averaged ones over the duration longer than the period of drift wave oscillation and shorter than the time scale of variation of the global density profile. The energy component of $(0,0)$ mode, which indicates that of the zonal flow, the sum of $(4,1)$ and $(5,1)$ modes, which form the streamer, and the net of the fluctuation energy are shown. It is found that when $\nu_{\text {in }}>0.05$, the zonal flow amplitude is small and the streamer modes are excited. As $\nu_{\text {in }}$ decreases, the zonal flow energy increases. The inflection point is given by $\nu_{\text {inc }} \sim 0.052$, where nonlinear structure changes drastically.

There are two dominant energy exchange paths from the $m \neq 0$ mode by mode coupling. One is the $(0,0)$ mode to form the zonal flow, and the other is the mediator mode [ $(1$, 2 ) in this case] to form the streamer. These two kinds of structural formation mechanisms are involved, but only one of the structures can appear in stationary states from their competitive nature. The streamer can be formed without the zonal flow formation. When the zonal flow is formed, the $\mathrm{E} \times \mathrm{B}$ shearing of the zonal flow breaks the phase locking of the modes, so the streamer is not formed, even though amplitudes of the modes are large. Figure 12(a) shows density profiles at the saturation phase with $\nu_{\text {in }}=0.02,0.04$, $0.05,0.08,0.1$. The case without the convective nonlinearity is also shown as "linear." Figure 12(b) shows the instantaneous linear growth rates evaluated with the density profile at the saturation phase. It is seen that the number of unstable modes dose not change in the streamer case with $\nu_{\text {in }}>\nu_{\text {inc }}$ $\sim 0.05$. On the other hand, it increases in the zonal flow case with $\nu_{\text {in }}<\nu_{\text {inc }}$, as $\nu_{\text {in }}$ decreases. Comparing a zonal flow case

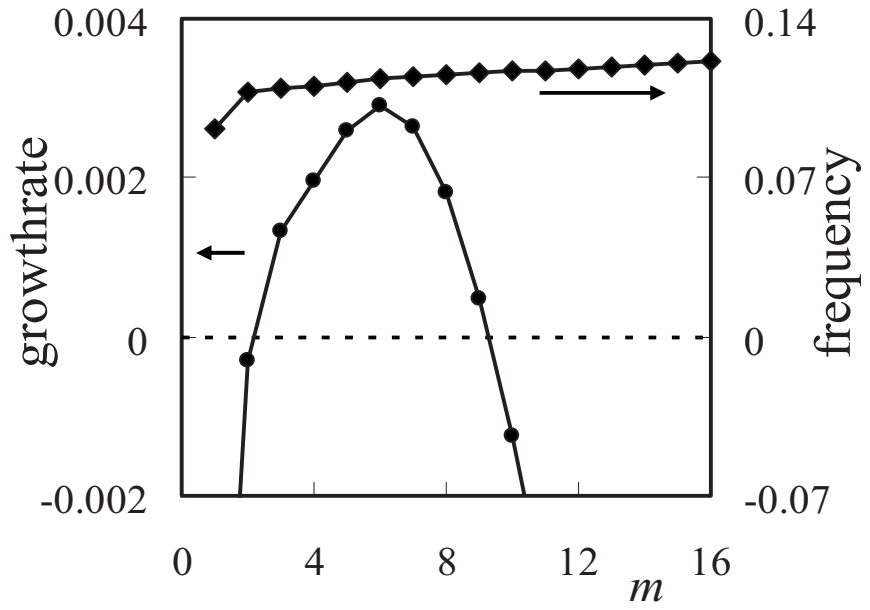

FIG. 8. Dependencies of the instantaneous linear growth rate and eigenfrequency on the azimuthal mode number $m$ in the case of $n=1$ and $\nu_{\text {in }}=0.1$. These growth rates are calculated using the density and potential profile at $t=8000$.

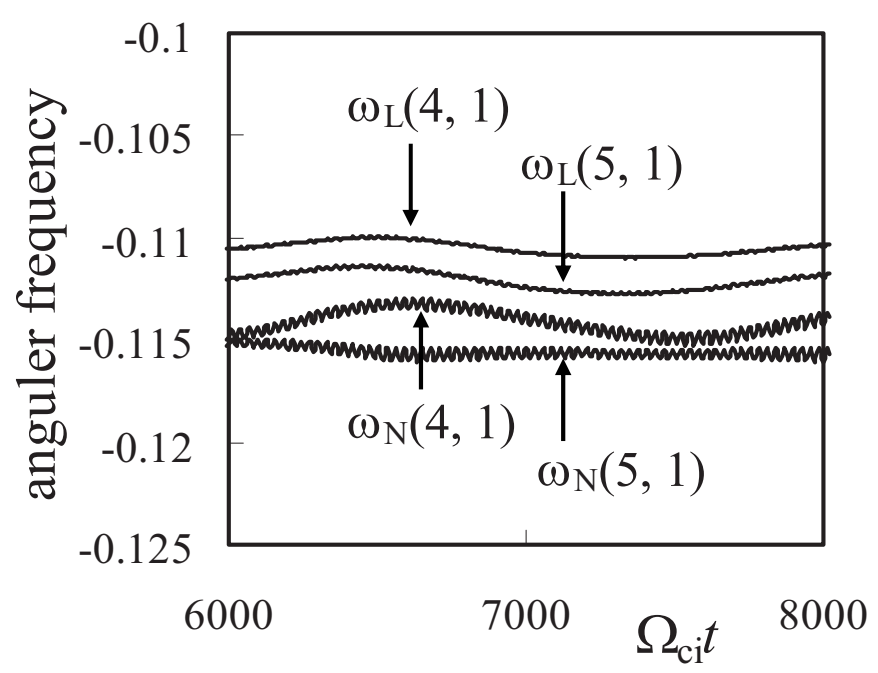

FIG. 9. Angular frequencies in the saturation phase $\omega_{N}$ and linear eigenfrequency $\omega_{L}$ of $(4,1)$ and $(5,1)$ modes. 
(a)

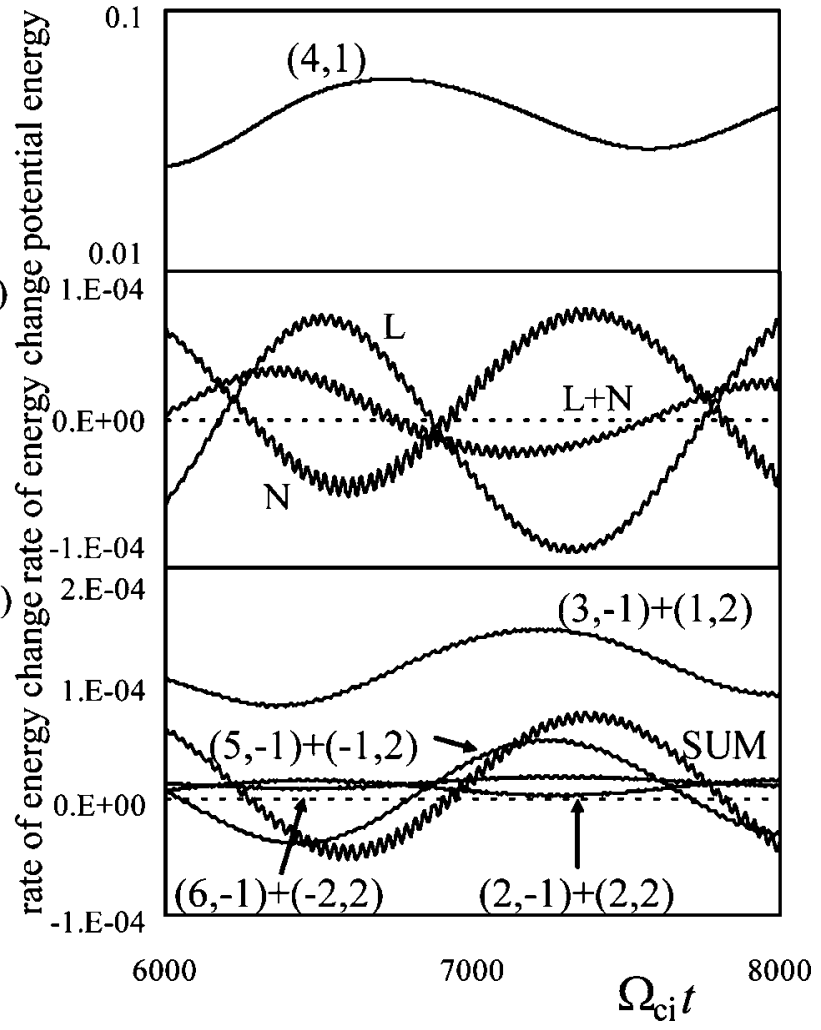

FIG. 10. (a) Time evolution of the electrostatic potential of the $(4,1)$ mode. (b) Rates of the energy transfer. The part proportional to the own mode energy (LT), the other nonlinear contribution (NT), and their sum (LT $+\mathrm{NT}$ ) are shown. (c) Rates of the nonlinear energy transfer decomposed into each three-wave coupling.

(a)

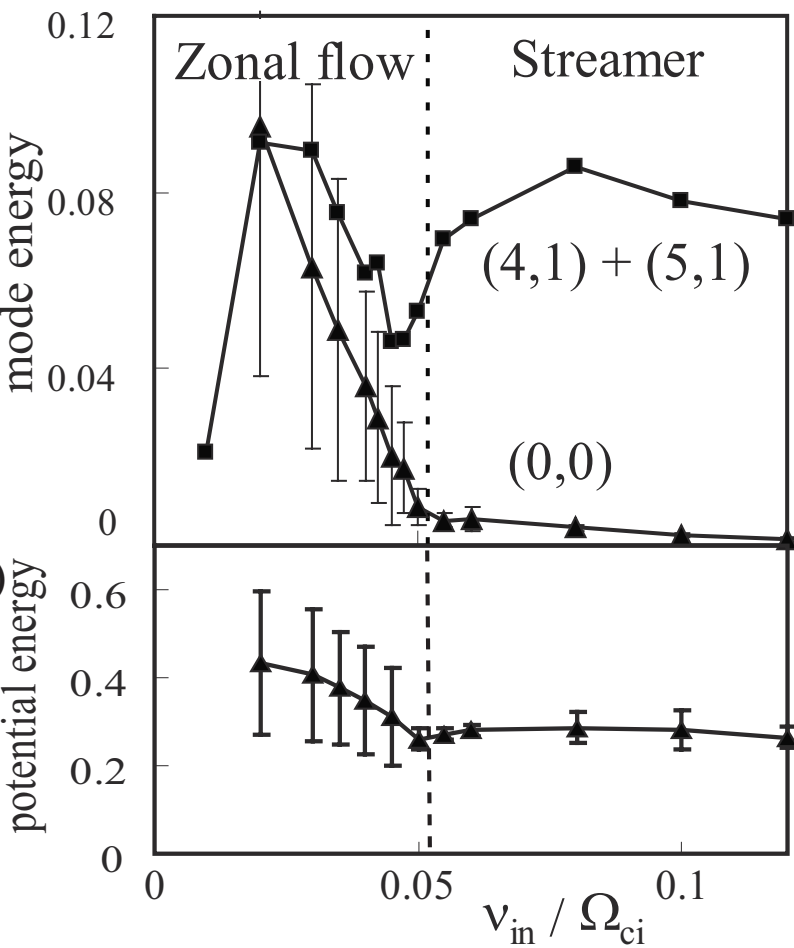

FIG. 11. Dependencies of the fluctuation energies of the electrostatic potential on $\nu_{\text {in }}$. Energies of $(a)(0,0)$ mode, the sum of $(4,1)$ and $(5,1)$ modes, and (b) net of fluctuations are shown.
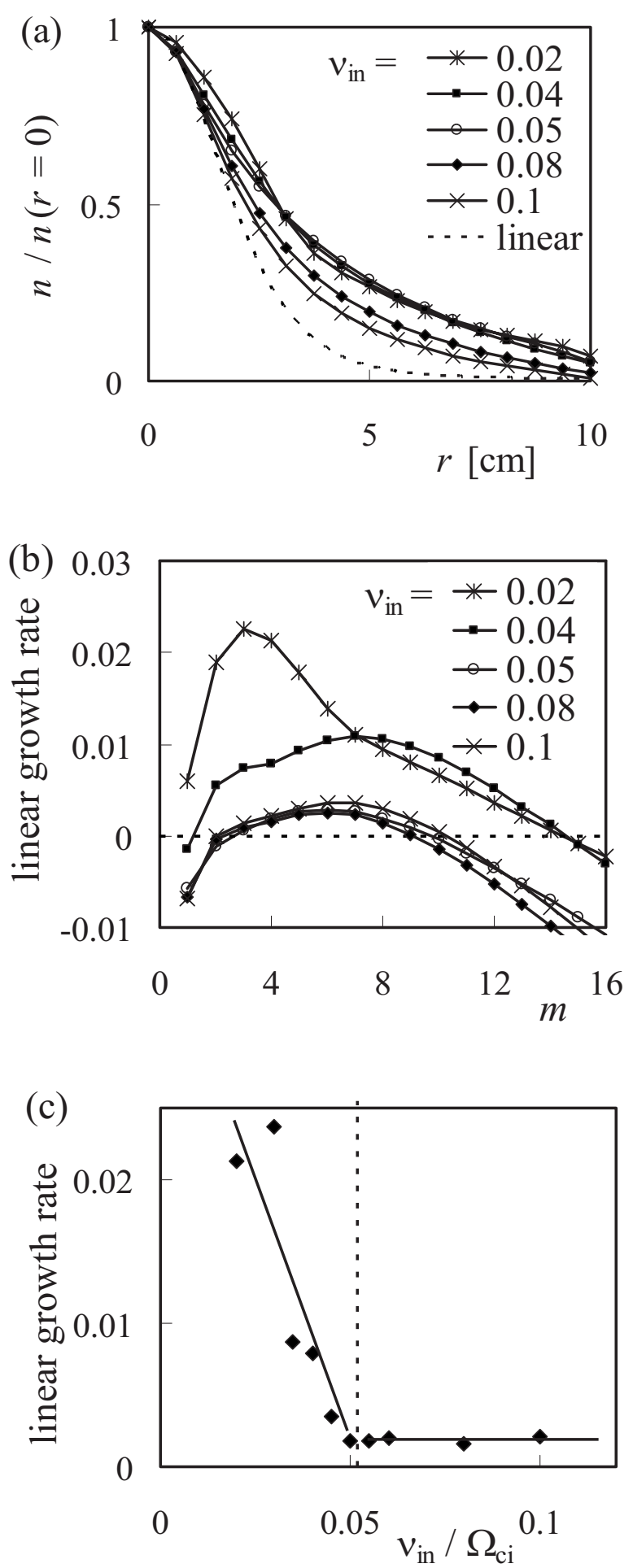

FIG. 12. (a) Density profiles at the saturation phase with $\nu_{\text {in }}$ $=0.02,0.04,0.05,0.08,0.1$. The densities are normalized by those at $r=0$. The dashed line shows the solution of the linearized equation of Eq. (1), which corresponds to the case without the convective nonlinearity. (b) Dependencies of the instantaneous linear growth rate calculated with density profiles in (a) on the azimuthal mode number, and (c) that of $(4,1)$ mode on $\nu_{\text {in }}$

with $\nu_{\text {in }}=0.04$ and streamer case with $\nu_{\text {in }}=0.06$, the $(6,1)$ mode has a maximum linear growth rate for both cases, but the absolute value of the instantaneous linear growth rate largely changes $\left[\gamma_{L}\left(\nu_{\text {in }}=0.04\right) / \gamma_{L}\left(\nu_{\text {in }}=0.06\right)=4.0, \quad\right.$ Fig. 12(c)]. The total fluctuation energy does not increase so 


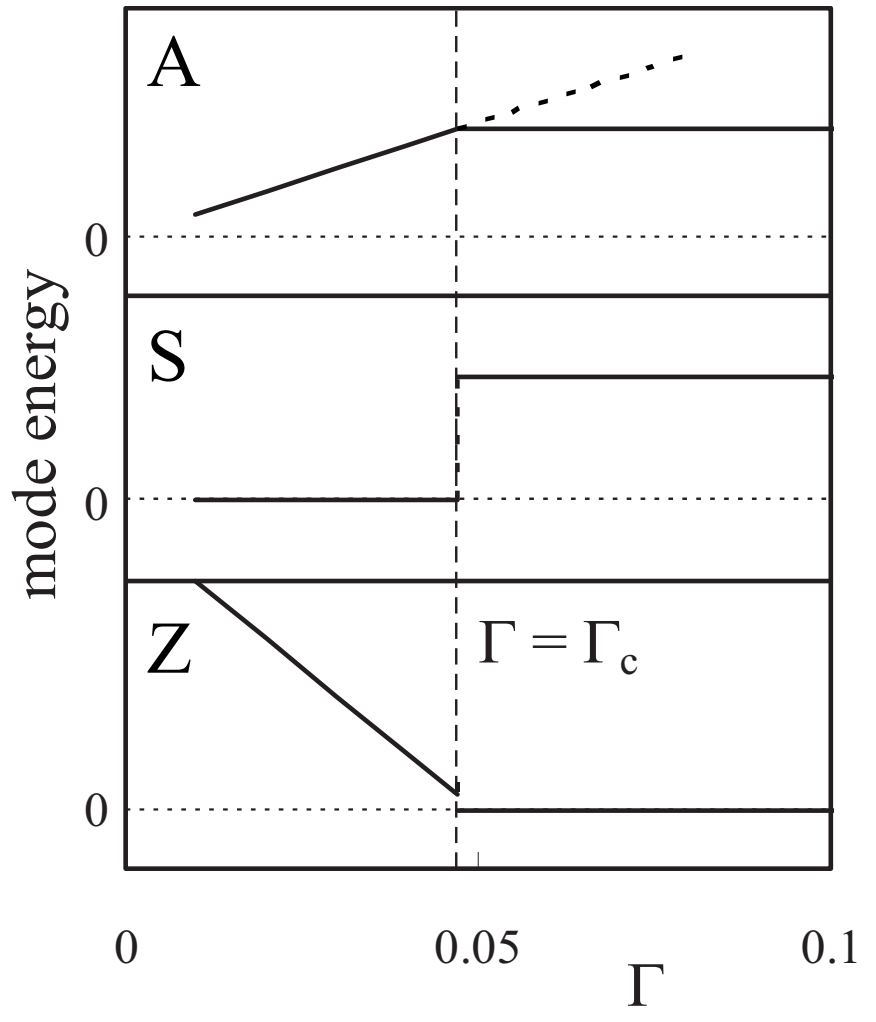

FIG. 13. Dependencies of mode energy $A, S$, and $Z$ on the zonal flow damping rate $\Gamma$, which is obtained from the set of Eqs. (6)-(8). Only $\Gamma$ is varied, and the other coefficients are fixed in the parameter scan. A transition takes place at $\Gamma=\Gamma_{\mathrm{c}}$.

much in accordance with the linear growth rate $\left[E_{\phi}\left(\nu_{\text {in }}\right.\right.$ $=0.04) / E_{\phi}\left(\nu_{\text {in }}=0.06\right)=1.2$, Fig. 11(b)]. On the other hand, the energy of the $(0,0)$ mode greatly changes $\left[E_{\phi}(0,0)\left(\nu_{\text {in }}\right.\right.$ $=0.04) / E_{\phi}(0,0)\left(\nu_{\text {in }}=0.06\right)=6.0$, Fig. 11(a)], which gives rise to the suppression of the fluctuations. This suggests that the selection of the turbulent structures is dictated by the damping of zonal flow by $\nu_{\text {in }}$ which self-regulates drift wave turbulence.

A simple analytical model including one unstable mode (drive) and two paths of mode coupling to a stable mode (sink) can give energy balance in three modes, ${ }^{24}$ and shows the selective excitation with the critical value of the zonal

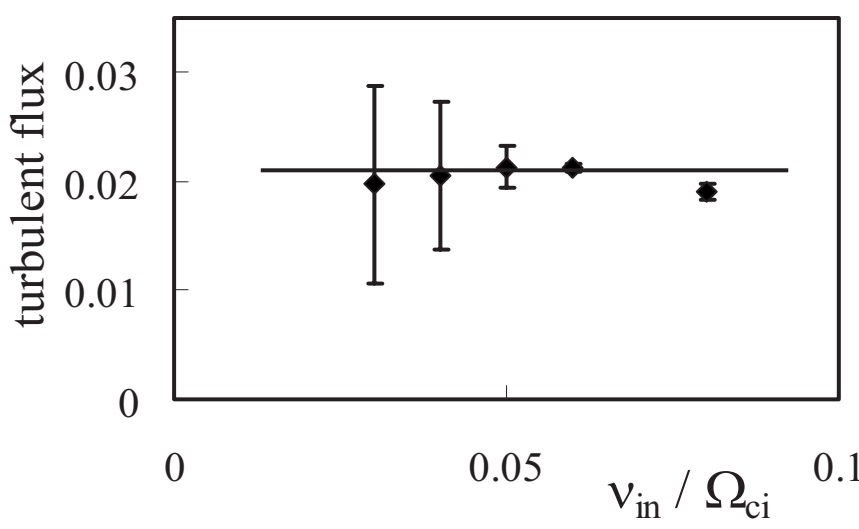

FIG. 14. Dependency of turbulent flux at $r / a=0.5$ on $\nu_{\text {in }}$.

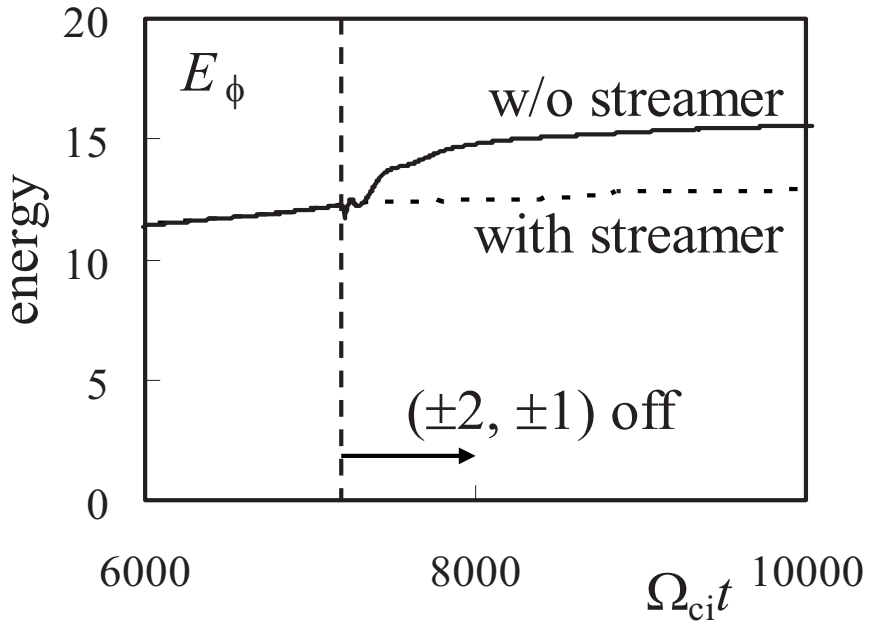

FIG. 15. Comparison of potential energy between the cases with and without streamer formation. The streamer does not sustain, when the mediate mode $(1,2)$ is artificially removed after the saturation in the case with $\nu_{\text {in }}$ $=0.1$.

flow damping rate. The set of model equations consists of the energy balance of source of instability $A$, mediator mode to form a streamer $S$, and zonal flow mode $Z$,

$$
\begin{aligned}
& \frac{\partial A}{\partial t}=\gamma_{L} A-A^{2}-\alpha A Z-\beta A S, \\
& \frac{\partial S}{\partial t}=-\gamma_{S} S+\beta A S-\alpha^{\prime} S Z,
\end{aligned}
$$

$\frac{\partial Z}{\partial t}=-\Gamma Z+\alpha A Z+\alpha^{\prime} S Z$

where $\gamma_{L}, \gamma_{S}$, and $\Gamma$ are linear growth rates (or damping rate) of the unstable mode, the zonal flow mode, and the mediator mode, respectively, and $\alpha, \beta$, and $\alpha^{\prime}$ are coupling coefficients with the other modes. The steady state solutions with

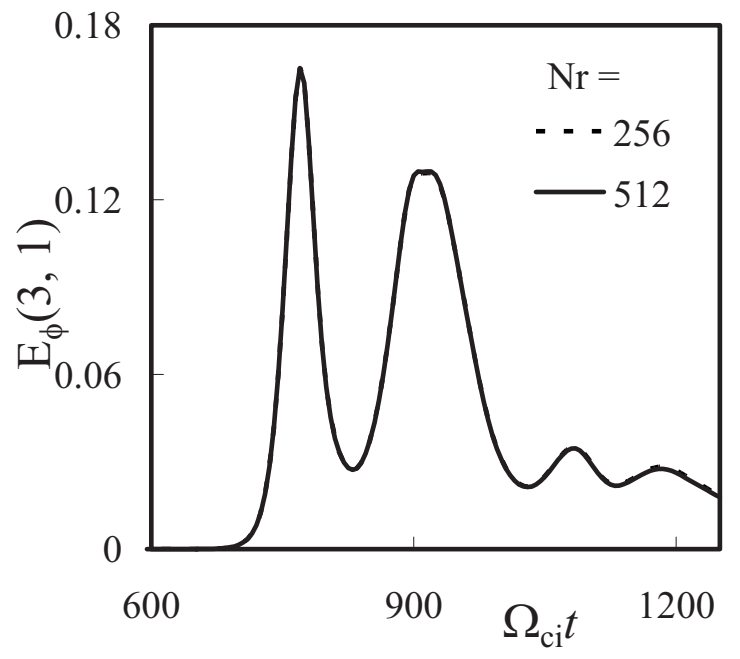

FIG. 16. Comparison of the time evolutions of the mode energies with different numbers of grids in the radial direction. Those are the cases with $\nu_{\text {in }}=0.02$, when the zonal flow is formed. Time evolutions in the two cases are indistinguishable. 

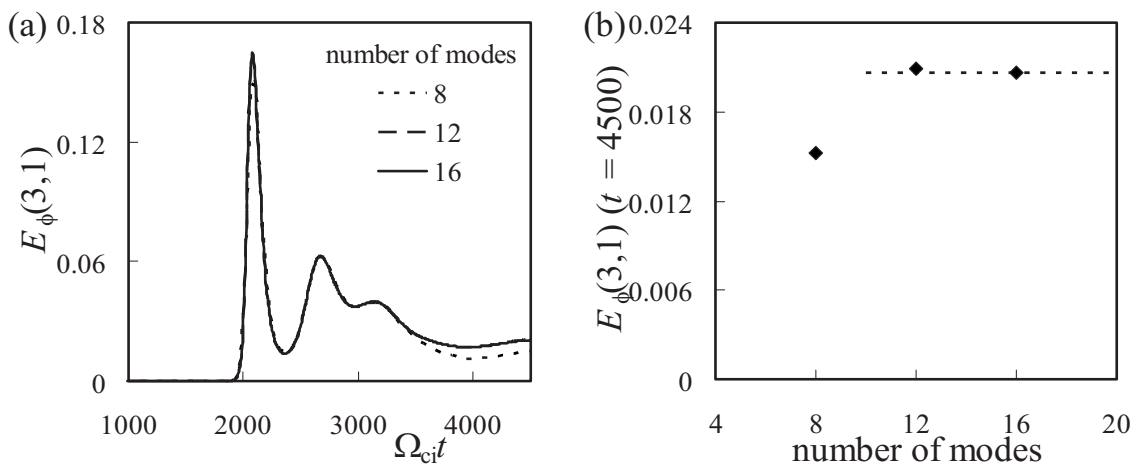

$\alpha^{\prime}=0$ are given as shown in Fig. 13. The zonal flow damping rate is used as a control parameter, and the critical value for structural bifurcation is obtained as

$$
\Gamma=\Gamma_{c}=\alpha \gamma_{s} / \beta .
$$

When the zonal flow damping rate is larger than $\Gamma_{c}$, only the streamer mode is excited. When the zonal flow damping rate is smaller than $\Gamma_{c}$, only the zonal flow mode is excited, and the energy increases linearly, as the zonal flow damping rate becomes small. The value of $\nu_{\text {in }}$ represents the strength of the zonal flow damping rate, on which the selection of the turbulent structures depends on.

In this way, the bifurcation of the structures in turbulent plasmas has been represented using the dictating parameter ( $\nu_{\text {in }}$ in this case). When $\nu_{\text {in }}$ is much smaller than $\nu_{\text {inc }}\left(\nu_{\text {in }}\right.$ $<0.02$ ), the mean density profile greatly changes [Fig. 12(a)]. Smaller $\nu_{\text {in }}$ destabilize the drift wave and the mode with maximum instantaneous linear growth rate changes, accordingly $\left[(3,1)\right.$ mode in the case with $\nu_{\text {in }}=0.02$, Fig. 12(b) $]$. The characteristic feature of the bifurcation is reproduced by the simple model in the neighbor of the transition boundary $\left(\nu_{\text {in }} \sim \nu_{\text {inc }}\right)$, although the stabilization effect on the drift wave due to $\nu_{\text {in }}$ give rise to some modification shown in Fig. 11.

In high-temperature plasmas as tokamak core plasmas, collisions with neutrals do not affect the turbulent structure, although they do in the edge region. An investigation of other control parameters, damping the zonal flow, is important to obtain the selection rule for turbulent structural formation in toroidal plasmas.

\section{EFFECT ON TRANSPORT}

It is well known that zonal flows suppress turbulent transport, and streamers induce convective transport. ${ }^{1}$ In the streamer case, the linear growth rate, and therefore, the fluctuation energy are small and constant, not depending on $\nu_{\text {in }}$ (Figs. 11 and 12). On the other hand, the linear growth rate and the fluctuation energy increase as $\nu_{\text {in }}$ decreases in the zonal flow case. These tendencies imply that the profile change in the zonal flow case greatly destabilizes the system and could induce a large turbulent particle flux. However, the particle source is fixed through the $\nu_{\text {in }}$ scan, so the turbulent fluxes have the same level, not depending on $\nu_{\text {in }}$, as shown in Fig. 14, which describes the dependency of the turbulent particle flux $\left\langle\tilde{N} \tilde{V}_{r}\right\rangle$ on $\nu_{\text {in }}$.
When $\nu_{\text {in }}$ is large, fluctuation amplitudes are rather small, so the turbulent conductive transport is small, but convective transport is generated by the streamer. We also carried out a simulation, in which the mediate mode $(1,2)$ is artificially removed after the saturation with $\nu_{\text {in }}=0.1$, when the streamer is formed with the $(1,2)$ mode. The streamer is not sustained and only a single mode becomes dominant without the $(1,2)$ mode. In this no streamer case, the fluctuation energy is $20 \%$ larger than those in the streamer case (Fig. 15), though the turbulent flux and density profiles are almost the same (the maximum of the difference of the density gradient is 5\%). That means the particle flux per the same fluctuation energy is larger in the streamer case. The discrepancy shows the drive of larger convective transport by the streamer. Energy condensation in the azimuthal direction by streamer formation enhances the radial transport in this case.

Qualitatively, the following pictures are drawn: When $\nu_{\text {in }}$ is large, fluctuation amplitudes are rather small, so the turbulent conductive transport is small, instead convective transport by the streamer plays a major role for transport. On the other hand, when $\nu_{\text {in }}$ is small, fluctuation amplitudes are large, however, the large turbulent transport is suppressed by the zonal flow and quasilinear density flattening. ${ }^{17}$ In this way, the particle transport is affected by the turbulent structures in the flux driven system.

\section{SUMMARY}

We have carried out the nonlinear simulation of the resistive drift wave in cylindrical plasmas, using the threedimensional turbulence simulation code NLD. Turbulence with a zonal flow or streamer was obtained in the nonlinear saturation stages. Nonlinear coupling between modes, which have neighboring $m$ and the frequency close to each other, forms a streamer. The streamer in our simulation is localized in the $\theta$ direction, and has a different time scale from that of the drift wave. The analysis of the energy transfer of each mode shows coupling between $(4,1)$ and $(5,1)$ modes by means of the mediator mode $(1,2)$ is important for the streamer formation. Simulations showed the selective formation of the zonal flow and streamer using $\nu_{\text {in }}$ as a control parameter. The value of $\nu_{\text {in }}$ represents the strength of the damping force of the turbulent structures, and if the collision frequency is large, the zonal flow remains stable, and the streamer is formed. The bifurcation of the formed structures 


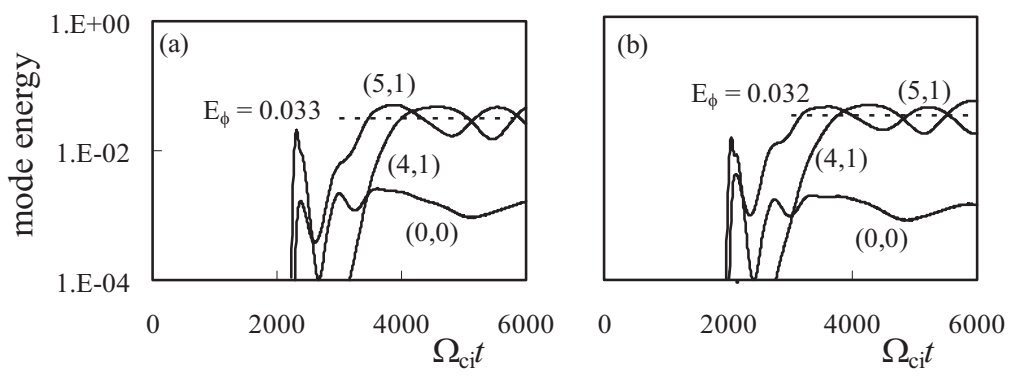

FIG. 18. Time evolutions of the mode energies with initial conditions of (a) the $( \pm 1, \pm 1)$ dominant case and (b) the same magnitude case. This is the case with $\nu_{\text {in }}=0.1$.

in turbulent plasmas has been obtained and their effects on particle transport have been revealed.

\section{ACKNOWLEDGMENTS}

The authors acknowledge discussions with Professor A. Fukuyama, Professor Y. Kawai, Dr. S. Shinohara, Dr. A. Fujisawa, Dr. Y. Nagashima, Dr. T. Yamada, Dr. S. Inagaki, Professor P. H. Diamond, and Professor G. R. Tynan.

This work is partly supported by a Grant-in-Aid for Specially Promoted Research (16002005) and for Scientific Research (19360418) of MEXT, by Research Fellowships of the Japan Society for the Promotion of Science for Young Scientists, and by the collaboration programs of NIFS (NIFS06KDAD005, NIFS07KOAP017, NIFS06KNXN079) and of RIAM of Kyushu University.

\section{APPENDIX: NUMERICAL CONVERGENCE}

Numerical accuracy has been verified by comparing calculations with different numbers of grids and Fourier modes. Simulations in this paper are performed with 256 grids in the radial direction. The relative error of the saturated mode energy is less than $3 \%$ with the case of 512 grids (Fig. 16), so the taken number of 256 grids is sufficient. In the azimuthal and axial directions, the Fourier expansion is adopted, and Fourier modes $(m, n)=(0,0)$ and $m= \pm 1- \pm 16, n$ $= \pm 1- \pm 16$ are kept $(m \times n=16 \times 16)$. Since modes with $(m, n)=(3-5,1)$ have the largest amplitudes, this number of modes should be kept at least for the numerical resolution. We also checked the case with $m \times n=8 \times 8$ and $12 \times 12$ (Fig. 17). The energy converges to a certain value according to the increase of the number of modes. The relative error of the mode energy is less than $2 \%$ between the cases with $m$ $\times n=12 \times 12$ and $16 \times 16$ in Fig. 17(a). Therefore, we conclude that the number of modes $m \times n=16 \times 16$ is tolerable in this case.

We also check the sensitivity to initial conditions using the different initial perturbations. Figure 18 shows the time evolution of mode energies in the case with $f_{1}=1$ $\times 10^{-8} \sin (\pi r / a)$ for $( \pm 1, \pm 1)$ modes and $f=f_{1} / 1000$ for the other modes. This is the case that only $( \pm 1, \pm 1)$ perturbations are dominant in the initial stage. At the earlier linear phase, the $(1,1)$ mode linearly grows at first, however, since the growth rates of $(2,1),(3,1)$ modes are larger than that of the $(1,1)$ mode, those modes overcome the $(1,1)$ mode at the latter linear phase. Finally, the same quasisteady state is attained, although the time for saturation is delayed compared to the case with the same amplitude for all modes. The relative error of the $(5,1)$ mode energy, averaged from $t=4000$ to 6000 , is only less than $5 \%$ for both cases.

${ }^{1}$ See reviews, e.g., P. H. Diamond, S.-I. Itoh, K. Itoh, and T. S. Hahm, Plasma Phys. Controlled Fusion 47, R35 (2005).

${ }^{2}$ A. Yoshizawa, S.-I. Itoh, and K. Itoh, Plasma and Fluid Turbulence (IOP, Bristol, 2003).

${ }^{3}$ W. Horton, Rev. Mod. Phys. 71, 735 (1999).

${ }^{4}$ Y. Saitou, A. Yonesu, S. Shinohara, M. V. Ignatenko, N. Kasuya, M. Kawaguchi, K. Terasaka, T. Nishijima, Y. Nagashima, Y. Kawai, M. Yagi, S.-I. Itoh, M. Azumi, and K. Itoh, Phys. Plasmas 14, 072301 (2007).

${ }^{5}$ G. R. Tynan, C. Holland, J. H. Yu, A. James, D. Nishijima, M. Shimada, and N. Taheri, Plasma Phys. Controlled Fusion 48, S51 (2006).

${ }^{6}$ F. Brochard, G. Bonhomme, E. Gravier, S. Oldenbürger, and M. Philipp, Phys. Plasmas 13, 052509 (2006).

${ }^{7}$ C. Schröder, O. Grulke, T. Klinger, and V. Naulin, Phys. Plasmas 12, 042103 (2005).

${ }^{8}$ V. Sokolov and A. K. Sen, Phys. Rev. Lett. 92, 165002 (2004).

${ }^{9}$ T. Kaneko, H. Tsunoyama, and R. Hatakeyama, Phys. Rev. Lett. 90, 125001 (2003).

${ }^{10}$ M. Koga and Y. Kawai, Phys. Plasmas 10, 650 (2003).

${ }^{11}$ M. Inutake, J. Plasma Fusion Res. 4, 75 (2001).

${ }^{12}$ M. Kono and M. Tanaka, Phys. Rev. Lett. 84, 4369 (2000).

${ }^{13}$ G. Y. Antar, J. H. Yu, and G. Tynan, Phys. Plasmas 14, 022301 (2007).

${ }^{14}$ T. Windisch, O. Grulke, and T. Klinger, Phys. Plasmas 13, 122303 (2006).

${ }^{15}$ K. Terasaka, S. Shinohara, Y. Nagashima, T. Yamada, M. Kawaguchi, T. Maruta, S. Inagaki, Y. Kawai, N. Kasuya, M. Yagi, A. Fujisawa, K. Itoh, and S.-I. Itoh, J. Plasma Fusion Res. 2, 031 (2007).

${ }^{16}$ F. Brochard, T. Windisch, O. Grulke, and T. Klinger, Phys. Plasmas 13, 122305 (2006).

${ }^{17}$ N. Kasuya, M. Yagi, M. Azumi, S.-I. Itoh, and K. Itoh, J. Phys. Soc. Jpn. 76, 044501 (2007).

${ }^{18}$ N. Kasuya, M. Yagi, and K. Itoh, J. Plasma Phys. 72, 957 (2006).

${ }^{19}$ G. N. Kervalishvili, R. Kleiber, R. Schneider, B. D. Scott, O. Grulke, and T. Windisch, Contrib. Plasma Phys. 46, 739 (2006).

${ }^{20}$ V. Naulin, O. E. Garcia, A. H. Nielsen, and J. Juul Rasmussen, Phys. Lett. A 321, 355 (2004).

${ }^{21}$ M. Ignatenko, M. Azumi, M. Yagi, S. Shinohara, S.-I. Itoh, and K. Itoh, Jpn. J. Appl. Phys., Part 1 46, 1680 (2007).

${ }^{22}$ C. Schröder, Ph.D. thesis, Ernst-Moritz-Arndt-University, Greifswald, 2003.

${ }^{23}$ M. Yagi, S.-I. Itoh, K. Itoh, M. Azumi, P. H. Diamond, A. Fukuyama, and T. Hayashi, J. Plasma Fusion Res. 2, 025 (2007).

${ }^{24}$ A. Das, A. Sen, S. Mahajan, and P. Kaw, Phys. Plasmas 8, 5104 (2001). 